\title{
Interactive comment on "Potential impact of aerosols on convective clouds revealed by Himawari-8 observations over different terrain types in eastern China" by Tianmeng Chen et al.
}

Tianmeng Chen et al.

zhanqing@umd.edu

Received and published: 4 February 2021

We thank the reviewers for their thoughtful and excellent comments and suggestions. We have tried as much as possible to address all concerns and have revised the manuscript accordingly. Please see the supplement file for the responses and the revised manuscript with track changes.

Please also note the supplement to this comment: 
Interactive comment 